\title{
Dispositivos estatales, ilegalismos y prácticas sociales en la frontera Brasil-Paraguay (1890-2015)
}

\author{
State devices, illegalisms and social practices in the \\ Brazil-Paraguay border (1890-2015)
}

\author{
Eric Cardin a* (iD) https://orcid.org/0000-0001-7813-5544 \\ Cintia Fiorotti Lima ${ }^{\text {b }}$ (i) https://orcid.org/0000-0003-2704-3230
}

${ }^{a}$ Universidad Estadual del Oeste de Paraná, Brasil, correo electrónico: eric_cardin@hotmail.com

b Secretaria do Estado de Educaçào do Paraná, Brasil, correo electrónico: cintiafiorotti@hotmail.com

\section{Resumen}

El objetivo de la investigación es analizar el desarrollo de las prácticas ilegales y sus relaciones con los aparatos de fiscalización y represión en la frontera Brasil-Paraguay, específicamente en los límites entre Foz de Iguazú-Ciudad del Este y Guaíra-Salto del Guairá. Metodológicamente, utilizamos entrevistas, fuentes bibliográficas y procesos criminales. A través del abordaje histórico sociológico, problematizamos las relaciones entre las prácticas ilegales y los aparatos estatales

Recibido el 13 de junio de 2017. Aceptado el 24 de mayo de 2018. Publicado el 21 de junio de 2018.

${ }^{*}$ Autor para correspondencia: Eric Cardin, correo electrónico: eric_cardin@hotmail.com

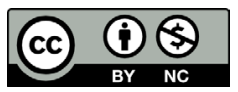

Esta obra está protegida bajo una Licencia Creative Commons Atribución-NoComercial 4.0 Internacional. en tres momentos específicos, que representan periodos en los cuales el gobierno brasileño y los habitantes de las zonas referidas presentaron posiciones diferenciadas en relación con las fronteras. La investigación permitió concluir que la organización del mercado de trabajo y las vivencias de los habitantes de la frontera están relacionados con la intensidad de la presencia del Estado en la región estudiada.

Palabras clave: frontera, Estado, sociedad, prácticas ilegales.

\section{Abstract}

The objective of the investigation is to analyze the development of illegal practices and their relations with the control and repression devices in the Brazil/ Paraguay border, specifically in the boundaries between Foz de Iguazú-Ciudad del Este and Guaíra-Salta del Guairá. Methodologically, we use interviews, bibliographic sources and criminal processes. Through the sociological historical approach, we problematize the relations between illegal practices and state apparatus in three specific moments, which represent periods where the Brazilian government and the inhabitants of the zones referred to presented different positions in relation to the borders. The investigation allowed to conclude that the organization of the labor market and the experiences of the residents of the border are related to the intensity of the presence of the State in the region studied.

Keywords: borders, State, society, illegal practices. 


\section{Introducción}

La frontera entre Brasil y Paraguay se presenta como una de las zonas limítrofes más dinámicas en América del Sur, lo que despierta el interés mediático y académico. En las últimas dos décadas muchos investigadores estudiaron la región y promocionaron diálogos con estudiosos de otras localidades, donde se destacan las relaciones establecidas con las investigaciones producidas en Argentina (Giménez y Montenegro, 2006; Montenegro y Giménez, 2010; Renoldi, 2014) y en México (Camal-Cheluja, Arriaga-Rodriguez y Cardin, 2015). Entre acercamientos y alejamientos, se destaca la mirada atenta atribuida a la compleja relación entre los Estados nación y la vida cotidiana de los trabajadores que viven los límites internacionales todos los días.

Cualquier observación menos atenta o descuidada de las fronteras latinoamericanas nos instiga de inmediato a la construcción de prejuicios que pueden tratar sobre el exceso de la presencia estatal, visualizada en la militarización de las fronteras, o, por el contrario, su ausencia, lo que genera el mito de la frontera sin ley. Sin embargo, las diferentes investigaciones realizadas en regiones de límites internacionales han demostrado que la realidad social existente en tales localidades es, en parte, derivada de las relaciones entre las acciones políticas promocionadas por los dispositivos estatales, que, en gran medida, visan reglamentar comportamientos y las prácticas sociales desarrollados por las poblaciones locales.

En este contexto, el objetivo del texto es reflexionar sobre el flujo transfronterizo en la región de confluencia de las fronteras de Brasil y Paraguay, observando las relaciones de estos flujos con los cambios en la forma de actuación del Estado brasileño en la frontera. Intentamos aprehender estas relaciones por medio de estudios bibliográficos y también utilizando algunas informaciones obtenidas en entrevistas orales y en registros de procesos criminales.

Históricamente, se observa que la circulación de trabajadores y mercancías entre Brasil, Paraguay y Argentina era común desde el siglo xIx (De Souza, 2009). En el pasado, aunque pudiera existir un reconocimiento de los límites territoriales, no había represión en relación con tales prácticas, aspecto fundamental para pensar cómo el comercio y el tránsito en la frontera no eran visualizados tan solo con base en los dispositivos jurídicos. Las restricciones en la circulación de personas y mercancías en la triple frontera se inician durante el primer gobierno de Getulio Vargas y se cristalizan en la dictadura civil-militar brasileña. A lo largo de este periodo, se constatan dos fenómenos fundamentales para la comprensión del rol que la frontera comienza a adquirir para el Estado: la necesidad de difusión de la brasilidad y el entendimiento que las fronteras son zonas de seguridad nacional, y por eso, lugares que requieren más atención con la consolidación del poblamiento y de la estructura urbana.

Por fin, con el proceso de democratización y reorganización de la economía mundial - expansión del modelo neoliberal, fortalecimiento de los bloques económicos y de los acuerdos bilaterales- el control aduanero en los límites de Brasil con Paraguay empieza a sufrir oscilaciones relacionadas con la importancia que la circulación de trabajadores y mercancías adquiere en la manutención del equilibrio del mercado interno brasileño. Dentro de diferentes coyunturas que marcan la relación de los Estados con sus límites territoriales, se observan modificaciones en las prácticas de los dispositivos de control y represión, visualizadas en la existencia de diferentes niveles de control y de tolerancia en relación con la circulación de personas y bienes. 


\section{Los límites invisibles y la circularidad en la frontera}

Hay un imaginario popular sobre los habitantes de la región de confluencia de la frontera Brasil-Paraguay que sostiene la idea de que entre ellos no había un claro reconocimiento de los límites internacionales hasta el comienzo del siglo xx. Los marcos jurídicos, aunque pudieran ya existir, cumplían un rol tan solo figurativo en aquellos paradores (Colodel, 1988; Lima, 2001; Sperança, 1992; Wachowicz, 1982). Los habitantes supuestamente transitaban como si las municipalidades fronterizas tuvieran una misma estructura y hubieran sido pensadas para garantizar la coexistencia de las diversas nacionalidades en un mismo espacio, compartiendo una logística que garantizaría el flujo comercial y cultural entre las diferentes naciones. Aunque eso pueda ser un poco exagerado, tal percepción no está totalmente equivocada.

Como nos recuerda Adriana Dorfman (2009, p. 2), una "buena frontera internacional debe materializarse en un discontinuo cultural", ya que, supuestamente, la construcción nacional involucra el reconocimiento de los elementos que serían propios de cada nación. El idioma y sus idiosincrasias locales, el folclore, las tradiciones y los mitos de fundación, distinguirían los pueblos y justificarían las demarcaciones. En este contexto, si estas demarcaciones fueran exitosas, encontraríamos en las márgenes de los territorios nacionales "un cambio abrupto de uno a otro pueblo, de una a otra lengua, de una a otra cultura, acompañando los límites de la soberanía estatal" (Dorfman, 2009, p. 2).

Sin embargo, la "buena frontera", que sería oriunda de la combinación de rompimientos políticos, naturales y culturales, no es fácilmente encontrada, pues raramente hay coincidencia en el nivel de contacto con tales dimensiones. En línea general, la frontera se caracteriza por contrastes en diferentes escalas, no habiendo una regla universal que posibilite una generalización sobre tales diferencias. Aunque, independientemente de los acercamientos y alejamientos existentes, la frontera "atrae personas que desean beneficiarse de las ventajas presentes en el lugar, en la forma de un costo de vida menor, mayores oportunidades de trabajo, acceso a servicios como salud, energía y telefonía” (Dorfman, 2009, p. 3).

La experiencia de vida en la frontera otorga a sus frecuentadores los instrumentos necesarios para articular las diferencias identificatorias, instrumentalizando las personas para que se vuelvan portadores/pasadores de los bienes simbólicos o materiales que expresan tales contradicciones y diferencias como un savoir passer (saber pasar) adquirido por los habitantes de la frontera, acostumbrados a accionar diferencias y semejanzas nacionales, lingüísticas, jurídicas, étnicas, económicas, religiosas que ora representan ventajas, ora reducción de tránsito o derechos. La frontera es el lugar en que las diferencias tienen cita marcada (Dorfman, 2009, p. 3).

En la región investigada, el savoir passer y el sentimiento de vivir en una frontera libre son derivados de un contexto histórico anterior a la presencia efectiva del Estado en los límites entre Brasil y Paraguay. Tal situación puede promover el equívoco de poner como única cosa las prácticas del contrabando y las experiencias de vivir en la frontera (Dorfman, 2009; Godinho, 2009). En síntesis, el concepto de cultura de contrabando intenta aprehender, en un único término, el proceso de naturalización 
del contrabando en una situación de aceptación explícita de los moradores locales, que le garantiza legitimidad, y de tolerancia estatal, debido su complacencia con la situación y su dificultad objetiva de fiscalizar. Esto no quiere decir que la población local es responsable de la vigilancia de la circulación en la frontera, muy al contrario. La experiencia secular de la circulación hace que los habitantes locales no reconozcan la necesidad de fiscalización, pues la movilidad transfronteriza corresponde a una práctica que históricamente forma parte del cotidiano de los habitantes.

El esfuerzo al intentar captar una realidad para insertarla en un modelo analítico deja escapar sutilezas. El hecho de "saber pasar" no permite que rotulemos de contrabandistas a todos aquellos que son poseedores de habilidad y tampoco nos permite generalizar la práctica para la totalidad de los moradores de las regiones de frontera. Como los demás hombres y mujeres, los sujetos sociales insertados en el contexto investigado trabajan, producen y habitan el lugar donde viven en un sentido amplio; sin embargo, la intervención y la utilización de espacio en la frontera, aunque sea intencional, no es calculada o pensada de modo estratégico, ella es vivida y modificada conforme los seres se relacionan con los materiales disponibles. En el intervenir nos formamos, no por el fin que anhelemos sino por los medios que desarrollemos.

La producción y la ocupación del espacio, y de la propia vida, modifican constantemente el haz de líneas trazadas por los sujetos, tejiendo la dimensión histórica, y "conforme se mueven juntos a través del tiempo y se encuentran unos a los otros, estos caminos se entrelazan para formar una inmensa y continua hilandería en evolución" (Ingold, 2015, p. 34). Pensar que los seres habitan y no simplemente construyen o producen el lugar en que están posee implicaciones analíticas, pues se destaca la ausencia de separación entre el sujeto y el medio. Cuando construimos o producimos el lugar en que vivimos, nosotros simplemente ocupamos una estructura, que podrá ser alterada de modo instrumental, hecho que nos permite observar la acción de un sujeto activo en un medio pasivo.

El haz de líneas, compuesto por hilos tejidos por los diferentes moradores, se ata en una gran trama, que se transforma a lo largo de la historia conforme las experiencias, las intencionalidades y el medio se relacionan. Pensar en una cultura de contrabando es pensar en el fin, o sea, en el "contrabando"; situación que se vuelve más complicada cuando reconocemos que el "saber pasar" tiene un origen anterior a la presencia efectiva del Estado nacional. Así, con el objetivo de problematizar la relación entre el "saber pasar" y el "contrabando", exploramos situaciones distintas donde se visualiza la "naturalización" del circular en la frontera y los intentos de disciplinar y controlar en diferentes periodos.

Hasta las primeras dos décadas del siglo xx, la presencia del gobierno brasileño y paraguayo en la región lindera entre los dos países era irrisoria, aunque existieran tratados internacionales posteriores a la Guerra de la Tríplice Alianza (1864-1870) en los que se establecían los límites internacionales y los hitos de tales fronteras. Aun reconociendo el imperio brasileño, en la figura de Don Pedro II, la importancia geopolítica de la localidad, la presencia más ostensiva de los dispositivos estatales brasileños comienza a realizarse después de 1930 a través de las políticas nacionales de poblamiento (Freitag, 2001). La consecuencia de esta ausencia es visualizada en la explotación descontrolada del territorio y por la gran presencia de extranjeros viviendo en la zona (Brito, 2005). 
Las publicaciones escritas y organizadas por memorialistas (Lima, 2001; Sperança, 1992) y por algunos historiadores de la década de 1980 (Colodel, 1988; Wachowicz, 1982), describen esta frontera de modo muy particular. Es común en tales registros el reconocimiento de que parte de la región este paraguaya y oeste brasileña eran explotadas por empresas extranjeras, conocidas como obrajes. En la gran mayoría actuaban en la extracción de recursos naturales, madera y yerba mate, utilizando, en gran medida, de fuerza de trabajo paraguaya (el mensu). La organización de estas empresas escapaba completamente del control paraguayo y brasileño. No había pago de tributos y ninguna protección laboral, originando una situación de explotación abusiva del medio ambiente y de los trabajadores.

Por lo tanto, es posible afirmar que la circulación de brasileños, paraguayos y argentinos por toda la región fronteriza en el final del siglo xIx y comienzo del xx, tenía como primer objetivo la explotación económica. La extracción de recursos naturales de un país para ser vendidos en otro, la complementación de renta por medio de la reventa de productos disponibles del otro lado de la frontera o la simple complementación de las mercancías necesarias en los hogares y comercios generaba flujos e interdependencia (Lima, 2001). Tales prácticas pueden ser entendidas como ilegalismos, ya que desde la fundación de la Colonia Militar de Foz do Iguazú en la desembocadura del Río Iguazú en 1888, ellas ya eran conocidas y toleradas por los dispositivos que deberían fiscalizarlas o cohibirlas.

Esta clase de acumulación primitiva de capital, donde la ausencia del Estado y cualquier hito regulatorio referente a la explotación económica de la región son aprovechados, se apropia de los saberes y de los habitantes locales. El conocimiento sobre el territorio y sobre sus potencialidades, la capacidad de desplazamiento y la adaptación a un medio ambiente — que para muchos podría ser hostil—, son elementos importantes para el mantenimiento de las prácticas de extracción realizadas hasta el comienzo del siglo xx. Por fin, la explotación denunciada por tales relatos señala la existencia de formas distintas de vivir en la frontera, donde se destacan los primeros intentos de "captura" de los modos de vivir de la población nativa.

La coexistencia de una gran circulación transfronteriza de trabajadores y mercancías y de un control permisivo por parte de los dispositivos del Estado brasileño también puede ser observada en las décadas de 1950 y 1960. En la investigación que realizamos en el acervo de autos criminales disponible en el Núcleo de Documentação Pesquisa (NDP/Unioeste), se constata la existencia de inquisiciones de supuestos crímenes de contrabando que terminan con la absolución de todos los acusados y el respectivo archivamiento, demostrando nuevamente la presencia del "saber pasar" y de los ilegalismos, confirmando que estos "no son ni disfunciones de las tecnologías políticas y tampoco excepciones de sus prácticas legales, sino parte constitutiva del ejercicio del gobierno" (Hirata, 2014, p. 98).

El proceso criminal 164/15 de 1961, por ejemplo, corresponde a un auto referente a una aprehensión realizada por la Policía Militar del Paraná. El documento retrata la situación de un trabajador paraguayo de nombre Rafael Barrios, que transportaba arroz, azúcar, aceite y combustible adquiridos en la municipalidad de Toledo, Paraná para ser entregados en la empresa en que trabajaba en Puerto Marangatú, Paraguay. Durante el recorrido fue interceptado y acusado de contrabando por los policías locales, pero absuelto por el Ministerio Público del Brasil. Según el fallo del juez responsable por el caso: 
Tal crimen no ha habido, pues muy bien esclarecido quedó que los indiciados no actuaban como contrabandistas, actuaban de buena fe, sin dolo, que es el elemento principal para la caracterización del delito de contrabando. El contrabando generalmente está realizado por una gran cantidad de mercancías y, lo que es importante y que caracteriza el crimen, es que él ha sido practicado a las escondidas, cuyos agentes buscan por todos los modos sustraerse a la vigilancia fiscal y policiaca. En el presente caso, el volumen de mercancías no autoriza tipificarlo como contrabando, ese no se destinaba a otro fin sino al del consumo de la empresa paraguaya compradora, que naturalmente se proveía de lo que podía en el Brasil, en Toledo, por ser un local de abastecimiento más cercano (Auto criminal, 1961).

El caso descrito gana relevancia por el hecho de que Barrios poseía en sus manos una carta de su "patrón" con la lista de productos para ser adquiridos en Brasil y también por haber comunicado al fiscal de la aduana brasileña que realizaría tales compras y, obtenido de este, una "autorización informal" para realizar los negocios. El trabajador, su "patrón" y el fiscal fueron acusados de estar involucrados en el "esquema" de contrabando, pero todos fueron absueltos. En la ocasión el juez admitió que tal práctica era necesaria y corriente en el cotidiano de la región. En esta situación queda la duda sobre la postura de los policías militares. ¿Ellos interceptaron al trabajador por no formar parte del supuesto "esquema" o interceptaron para ejercer la autoridad?

Según Cintia Fiorotti (2015), no era común una aprehensión de esta clase de mercancía transportada de modo no reglamentada entre Brasil-Paraguay y viceversa. Sin embargo, eso no quiere decir que no ocurría. El caso presentado señala que el transporte de alimentos y combustible en la frontera existía y era tolerado por algunos agentes del Estado (Fiorotti, 2015). Durante el levantamiento realizado no encontramos autos criminales de prisión de trabajadores que transportaban mercancías industrializadas destinadas para el propio consumo. Tal constatación es reforzada cuando observamos que tales prácticas todavía son realizadas, no obstante, la dirección de los flujos de las mercancías y sus especificidades varían de acuerdo con la estacionalidad y el cambio.

Este contexto, que denunciaría una imprecisión en cuanto a la definición y a la reglamentación de los usos y costumbres en la región fronteriza, también puede ser visualizada en otras fuentes y en periodos históricos más recientes. Entrevistas realizadas durante la investigación expresan situaciones que podrían ser consideradas curiosas. En el inicio del 2015, buscando interlocutores paraguayos que vivían en Guaíra, Paraná, Brasil, para constituir una red de contactos para una futura investigación, conversamos con muchas personas y ellas nos indicaron otras, supuestamente paraguayas y que vivían hace más tiempo en la ciudad. Entre estos interlocutores, las conversaciones establecidas fueron señalando la existencia de experiencias de vivir en la frontera compuesta por innumerables hilos tejidos en un conjunto de desplazamientos realizados a lo largo de diferentes trayectorias. En las narrativas hay saberes adquiridos durante los movimientos realizados, saberes que no son precisos o fundamentados, sino oriundos del propio vivir y que van siendo expuestos en las hablas, en los gestos y en las descripciones realizadas de los diferentes caminos transitados. Como destaca Ingold (2015), habitar, desplazar, conocer y describir hace parte de un mismo movimiento, que se sintetiza en el vivir. 
En este sentido, el pensamiento cartesiano no aprehende y tampoco explica las vidas de estos trabajadores y no hace parte de los mecanismos utilizados por los mismos para direccionamiento de sus prácticas, para mediar sus relaciones con el entorno y, mucho menos, para construir sus narrativas sobre las propias vidas. En los comentarios de los entrevistados, las nociones de espacio-tiempo y las propias identidades hacen parte de un mismo acto, cualquier intento de organización lineal de las trayectorias parte del esfuerzo por conducir la entrevista y no de la percepción de vida existente entre los diferentes interlocutores.

Las historias de Domingas Candi Lopes, de Maria Tereza Cabrera y de la familia Blas, por ejemplo, señalan un desplazamiento entre identidad física-jurídica y la supuesta identidad simbólica. La primera interlocutora, que tenía 95 años en la ocasión, era identificada como paraguaya por todos los miembros de la comunidad de migrantes de la municipalidad de Guaíra, Brasil, menos por ella propia. En "portuñol" relató sobre el origen paraguayo y de toda su familia, expuso sobre el trabajo de extracción de madera y yerba mate realizado por los padres en mediados del siglo xx y afirmó haber nacido en el Mato Grosso, Brasil.

La segunda interlocutora, Maria Tereza Cabrera, quien tenía 54 años al momento de la entrevista, también trasmite el mismo desplazamiento entre las identidades. La entrevista fue realizada mientras ella hacía chipa, un saladito de almidón de mandioca típico de Paraguay, en la cocina de uno de los puntos tradicionales del encuentro de migrantes en Guaíra, donde hay una capilla de Nuestra Señora de Guadalupe. Inicialmente, hablando en portugués, la interlocutora afirmó ser paraguaya y nos narró su trayectoria de vida. Sin embargo, cuando ella explicó su actual ocupación en el Consulado brasileño en el Paraguay, empezó a hablar español y a reconocer su nacionalidad brasileña.

En el tercer caso, se visualiza la movilidad de la familia Blas acompañando el movimiento migratorio de brasileños y brasileñas hacia el Paraguay, estimulado por políticas gubernamentales paraguayas durante la década de 1970 (Albuquerque, 2010). Ana María se trasladó al país vecino y allá constituyó la familia, tuvo tres hijos que fueron criados con familias paraguayas y alfabetizados en español y en guaraní. Hace menos de dos años resolvieron trasladarse a Brasil e insertarse en el mercado de trabajo del país, laborando en madereras y en la cosecha de yuca, actividades que en la región son asociadas con los paraguayos. Sin embargo, aun con este perfil, la familia defiende de modo explícito una supuesta identidad brasileña.

En este momento no es nuestro objetivo discutir propiamente el contenido de las entrevistas y presentar detalladamente las narrativas, sino destacar solamente dos aspectos que fortalecen la idea del desencuentro de las fracturas natural, económica, política y cultural, que supuestamente definirían el proceso de demarcación de aquello que podría ser considerado una "buena frontera". Los casos presentados trasparecen la existencia de una clase de migración circular, donde los moradores transitan por los países flexionando sus propias identidades y, como consecuencia de esto, desarrollan redes trasnacionales que garantizan la permanencia de determinadas conexiones, de tramas.

El propósito de presentar tales casos es demostrar que, aunque la frontera sea explícita y que existan límites jurídicos entre las naciones y entre estos sujetos, la circulación y pertenencia no observa tales elementos, ser paraguayo o ser brasileño es circunstancial y el modo que las otras personas los identifican no necesariamente corresponde a la autoidentificación. Para estas personas, un mayor control aduanero 
es un obstáculo para la construcción cotidiana de una identidad híbrida (García, 1990), pues, como observa Grimson (2005), las políticas estatales para las fronteras no reflejan las experiencias de los sujetos fronterizos, sino solamente de las personas insertas de modo privilegiado en el mercado.

Los interlocutores señalan que para la práctica de desplazamiento, donde la frontera no se presenta como un impedimento, la relación que ellos establecen con los límites y con las propias identidades son construidas durante el vivir. Llevando eso en consideración, se vuelven más comprensibles las decisiones del Ministerio Público del Brasil que absolvían a los habitantes de la región cuando estos eran acusados de la práctica de contrabando durante las décadas de 1950 y 1970, y también el entendimiento de la dificultad en la construcción de la brasilidad o de la imposición de una soberanía en la región dentro de un periodo en que los dispositivos estatales actuaban de modo aún muy incipiente.

La situación de vivir la y vivir en la frontera no es compuesta por una historia lineal u homogénea. La Guerra de La Triple Alianza establecida por Brasil, Argentina y Paraguay llamó la atención de los gobiernos en relación con la geopolítica local, pero eso no representa cambios sustanciales en los flujos fronterizos existentes en la zona. Hasta la década de 1930, la región investigada sufría con un proceso intenso de expropiación de las fuerzas productivas, constituyendo un momento de acumulación primitiva de las familias que paulatinamente se vinculaban al sector turístico y hotelero y buscaron alejarse de los habitantes locales que poseían sus modos de vivir directamente relacionados con las posibilidades oriundas de la frontera.

Sin embargo, la coexistencia de diferentes usos de la frontera, marcada por la presencia de empresas de extracción de recursos naturales que explotaban a fuerza de trabajo local, de trabajadores que vivían de la agricultura de subsistencia y, al mismo tiempo, de "saber pasar", que servía de complementación de renta, no representa un momento o una situación pasada. Aunque los procesos productivos puedan haber cambiado, todavía hoy se visualiza la presencia de los diferentes usos de la frontera y también de los esfuerzos en reglamentar los usos y costumbres existentes. La diferencia se encuentra en la diversificación de las formas de acumulación de capital y en la sofisticación de las formas de disciplinarización.

\section{La frontera y los límites nacionales}

Los límites jurídicos intangibles y la circularidad de los habitantes de la región marcada por la frontera de Brasil con Paraguay, paulatinamente van siendo alteradas y disciplinadas por medio de dos movimientos políticamente más sistematizados que tenían el objetivo de garantizar la soberanía nacional y el control de la zona. El primero de ellos ocurrió durante la dictadura de Getulio Vargas cuando fueron creados nuevos estados-territorios en el país; uno de ellos fue denominado Territorio del Iguazú, que alcanzaba parte de los Estados de Paraná y de Santa Catarina. Las principales justificaciones para el emprendimiento fueron la necesidad de centralizar un núcleo de administración estatal en la región para facilitar las relaciones políticas con el gobierno federal y también garantizar el fortalecimiento de una identidad nacional. 
Se visualizan en este momento histórico dos elementos que están directamente correlacionados. La preocupación del gobierno brasileño sobre la frontera no estaba vinculada directamente a la fuga de divisas o a la entrada de mercancías consideradas ilegales en Brasil, sino a la necesidad de garantizar el fortalecimiento de una frontera étnica en relación con los otros países latinoamericanos. Observando el acervo de procesos criminales investigados, se destaca que el control de la circulación de mercancías, principalmente de los camiones que transportaban café, se inicia de modo más efectivo durante la década de 1950, o sea, en el periodo en que la región norte del estado del Paraná se presentaba como una gran productora.

Inicialmente, el objetivo del gobierno brasileño era garantizar que el territorio de la franja de frontera de Brasil con Paraguay fuera explotado por brasileños y desarrollar el sentimiento de pertenencia a la nación. Como fue dicho, hasta las primeras décadas del siglo xx, la movilidad de la población fronteriza era alta, dificultando la constitución de "identidades nacionales" y ocultando la existencia de los límites internacionales. A partir del gobierno varguista se busca la inversión de estas relaciones, explicitando la existencia de la frontera para inviabilizar el contínum cultural existente entre la población fronteriza. Si en el primer momento las fronteras eran ocultas, en el segundo se anhela la invisibilidad de las redes sociales trasnacionales que garantizan la circularidad de los habitantes locales.

Esta situación nos acerca a uno de los malentendidos existentes sobre las regiones de frontera. El establecimiento de la "buena frontera" es efectivo cuando el límite demarcado separa grupos que teóricamente serían distintos en su formación y en sus prácticas culturales. Sin embargo, tal "fractura" es inexistente en las localidades que sufrieron y aún sufren un contínuum de colononización (Dorfman, 2009). La existencia de una "buena frontera" haría posible la identificación de los modos de vivir y de las prácticas económicas y políticas que caracterizaría a cada nación; sin embargo, en una región de intenso flujo e interdependencia, el esfuerzo de precisar una "identidad nacional" es ignominioso y exige políticas sistemáticas de disciplinarización.

La constitución de un Estado nación pasa por la formación de una "identidad nacional”, o sea, por una homogenización de determinadas características culturales que supuestamente garantizarían la cohesión social en todo el territorio nacional. De este modo, la "política civilizatoria" que procura garantizar la brasilidad (Garcia, 1999), la chinelización (Valdebenito y Lube, 2014) o la argentinización (Zaidenwerg, 2013), se vuelve corriente en Estados relativamente nuevos. El intento de constituir el Territorio del Iguazú durante la primera gestión del presidente brasileño Getulio Vargas tenía esta finalidad, acercar a una población aislada del centro político del país, garantizando su reconocimiento y el desarrollo de un sentimiento de pertenencia a la nación, pero este objetivo no fue alcanzando de manera plena. Entre 1943 y 1946, se crearon y disolvieron seis diferentes territorios nacionales con el objetivo de proteger las fronteras nacionales durante la Segunda Guerra Mundial. En el caso del Territorio del Iguazú el esfuerzo en fortalecer la identidad nacional estuvo más relacionada con la nacionalización de la fuerza de trabajo en la década de 1930 por medio de la sustitución de los paraguayos y argentinos por brasileños.

En todos los casos, la cuestión de la identidad nacional en la región de confluencia de la frontera de Brasil, Argentina y Paraguay no es simple. Marcada por intensos flujos transnacionales a lo largo de toda la historia, la presencia de una "identidad pura", que supuestamente represente los estereotipos nacionales, es casi inexistente. 
La frontera se impone como un elemento que flexibiliza las estrategias y las propias identidades. Como destaca Néstor García Canclini (2000), lo que caracteriza la ciudad de frontera no es inmediatamente la presencia constante y arraigada de una identidad nacional, sino la posibilidad de la frontera, configurarse ora como un lugar de conexión ora de separación. George Simmel (1996) afirma que "a cada instante somos nosotros que separamos lo que está conectado o volvemos a unir lo que está separado" (Simmel, 1996, p. 10) No basta a los hombres fijar el camino entre dos lugares, hacemos hincapié por grabar "visiblemente el camino sobre la tierra para que estos lugares pudieran ser conectados nuevamente" (Simmel, 1996, p. 10). En este sentido, la construcción de puentes sería un ejemplo.

Construir un puente es materializar algo que existe en nuestras necesidades. No unimos algo si no reconocemos la existencia de la separación, sea impuesta por la geografía, sea impuesta por la historia de los hombres. Sin embargo, tal vez lo más importante sea el hecho de que no unimos algo si no lo deseamos. Pensar un puente como una metáfora de la frontera significa reconocer su potencialidad de garantizar o posibilitar la conexión o la reunión de lugares que, para hombres y mujeres, se encuentran separados. De este modo, el puente "supera el alejamiento de sus extremidades al mismo tiempo que lo vuelve perceptible y mensurable" (Simmel, 1996, p. 12). No obstante, el puente, que puede ser visualizado y pensado como una analogía de la frontera, muchas veces es insuficiente para representar la frontera.

El fortalecimiento de los dispositivos estatales en los límites del Brasil con el Paraguay refuerza otro carácter de la frontera, marcado por el control de los flujos. Así, si la frontera puede ser vista como un puente, ella también necesita ser pensada como una puerta administrada por los respectivos Estados nación. Mientras el puente se configura como un pasillo de conexión y de paso entre dos puntos, pasando una supuesta seguridad referente a la circulación, la puerta se constituye como una posibilidad de tránsito. El puente se mezcla con la naturaleza y con ella es naturalizada, volviéndose parte del paisaje.

Si en el primer momento se visualiza el esfuerzo de los habitantes de la frontera Brasil-Paraguay direccionada a la construcción de puentes, por no admitir la separación geográfica, política, por la búsqueda del fortalecimiento de sus redes sociales trasnacionales o por acreditar en la importancia de la libre circulación de los trabajadores y mercancías, a partir de determinado momento, con la presencia más efectiva del Estado, la preocupación es direccionada para la construcción de puertas. En otras palabras, las políticas desarrolladas durante el gobierno varguista buscaban fortalecer los mecanismos de control de flujos, estableciendo "puertas" para regular el tránsito en la frontera del Brasil con Paraguay.

El proceso de expansión de la frontera agrícola impulsada por los gobiernos brasileño y paraguayo que, en cierto modo, se ha facilitado con la construcción de la infraestructura de paso, explicita al mismo tiempo una especie de control. El Puente de la Amistad, los puestos aduaneros, estructuras que aparentemente vinculan a los dos países, en realidad sirven para restringir el paso y facilitar el control de las personas que pueden pasar. La política de ocupación agrícola, que posibilitó que miles de brasileños migraran a Paraguay, no era universal a todos los interesados, se limitaba a un perfil de trabajadores que era del interés de los gobiernos brasileño y paraguayo. En otros términos, el puente es, al mismo tiempo, estructura de aproximación y de control. 
El segundo movimiento de fortalecimiento de los dispositivos estatales es promocionado durante la dictadura civil-militar brasileña (1964-1985). En este periodo se constatan cambios importantes en la política de la frontera del país. En primer lugar, se visualiza que el intento de ocupación y control de las regiones limítrofes del país dejar de ser por medio de la construcción de una supuesta brasilidad y pasa a ser, de modo más efectivo, militar. Mientras que los esfuerzos de constitución de una Colonia Militar en la desembocadura del Río Iguazú en el final del siglo xix no obtuvo éxito debido a la aproximación de los militares a las prácticas cotidianas en los primeros moradores de la región (Lima, 2001), el segundo intento de ocupación militar fue más jerarquizada, impositiva e influente en las relaciones entre Brasil y Paraguay. ${ }^{1}$

La constitución otorgada en 1967 atribuía al Consejo de Seguridad Nacional la responsabilidad por las cuestiones relacionadas al desarrollo y la seguridad en los límites del territorio brasileño, posición que fue reforzada por la Ley n ${ }^{\circ}$ 6634/1979. En la ocasión se determinó: que el límite de las tierras públicas para alienación o concesión en la franja de frontera pasaba de 2000 para 3000 hectáreas; que la Federación dejaría de concurrir con $50 \%$ del costo de obras públicas municipales en la franja, pero se reservaría el derecho de concurrir con el costo total o parcial de acuerdo con el interés del área para la seguridad nacional; y, por fin, que el Consejo de Seguridad Nacional asumiría la responsabilidad por providencia de la consignación de los recursos para los proyectos en la franja de la frontera (Cardin, 2012).

El fortalecimiento de una ideología que mezclaba seguridad y desarrollo, es confirmado en la construcción de la Usina Hidroeléctrica de Itaipú Binacional, ubicada en la frontera de Brasil con Paraguay. Un proyecto de infraestructura que lograba resolver cuatro problemas enfrentados por el gobierno militar: 1) el Litigio de la "Sete Quedas", sobre la demarcación territorial en la frontera Brasil-Paraguay; 2) conducía el Paraguay para la influencia brasileña y, prácticamente, poniéndolo en una situación de "Estado Tampón" (Arriaga-Rodríguez y Visintin, 2012); 3) garantizaría una capacidad de suministro de energía suficiente para atender a las necesidades de las multinacionales interesadas en actuar en el país y, 4) definía el control estratégico de la región marcada por la confluencia de las fronteras de Brasil, Paraguay y Argentina (De Souza, 2011; Espósito, 2013;).

Dentro de un contexto general, el acuerdo binacional que ha posibilitado la construcción de la hidroeléctrica en el Río Paraná ha modificado la geopolítica del Conesur y, en una perspectiva local, transformó completamente la frontera entre los dos países. A partir de mediados de la década de 1970 las ciudades de Foz de IguazúBrasil y Ciudad del Este-Paraguay sufrieron inversiones en la estructura urbana para atender los miles de trabajadores que llegaban para actuar en las obras de la usina. Viviendas, mercados, escuelas, clubes, calles y avenidas fueron ejecutadas rápidamente para atender específicamente a los trabajadores fijados por Itaipú, rediseñando la geografía urbana y promocionando una explosión demográfica que comprometió la atención a los demás moradores de la región.

\footnotetext{
${ }^{1}$ No podemos dejar de destacar que en el mismo periodo la dictadura paraguaya del General Stroessner (1954-1989) también tuvo una importancia fundamental en la reorganización de la frontera, sea por haber oportunizado la entrada de más de 500000 brasileños al este de Paraguay o por la fundación de Ciudad del Este y la creación de la zona de tributación diferenciada.
} 
De inmediato, el intenso flujo migratorio ocurrido durante las décadas de 1970 y 1980 reforzaron problemas ya existentes relacionados con la falta de vivienda empleos, dificultades en el abastecimiento y en los índices de violencia, problemas que fomentaron una inflación artificial en la región derivada de la asociación de la gran búsqueda por innumerables tipos de mercancías y la escasez de las mismas. La construcción de la usina promovió un choque entre modos de vivir, entre ritmos temporales y espaciales que fueron juzgados de diferente forma por la población local (Catta, 2002). Además, alteró la historia de las municipalidades fronterizas de Guaíra y Salto de Guairá al sumergir las "Sete Quedas" durante la formación del Lago de Itaipu, extinguiendo así el principal atractivo turístico de la zona (Fiorotti, 2009).

El gran flujo migratorio atraído por la construcción de la hidroeléctrica, que posibilitó que la municipalidad de Foz de Iguazú, Brasil, obtuviera un aumento de más de 400\% de la población en tan solo diez años, lo que radicalizó los problemas existentes. La falta de vivienda y de empleo, agravada por los problemas de la estructura existente para la atención en las áreas de salud y educación, exigió cambios en las prácticas sociales de la población local, que se volvió más dependiente de la economía de los países vecinos (Cardin, 2015; Catta, 2002). No suficiente también modificó las relaciones de poder hasta entonces existentes (Ribeiro, 2015).

Durante las primeras décadas del siglo $\mathrm{xx}$, paulatinamente el poder económico y político quedó concentrado en los grupos que controlaban, primeramente, las actividades extractivas, y, posteriormente, el sector turístico. Sin embargo, con el inicio de la dictadura civil militar en 1964, los intendentes municipales empezaron a ser nombrados por el régimen, no teniendo vínculos con los habitantes locales. Tal situación promovió la importancia de la Usina de Itaipú en la ciudad que, además de ser la principal generadora de empleo y renta de la municipalidad, también se convirtió en un importante agente político, influenciando directamente las medidas desarrolladas en la década de 1970 (Ribeiro, 2015).

No coincidentemente, es en este mismo periodo que el mercado del Presidente Stroessner (actual Ciudad del Este) comienza a ganar expresión y a ocupar un lugar de destaque en la venta de productos norteamericanos y asiáticos. Creada en la década de 1960, la zona libre de comercio paraguayo empezó efectivamente a influenciar la economía de la región en la década posterior, en un momento de altas tasas de desempleo, derivadas, principalmente, de los cambios traídos por la hidroeléctrica (Cardin, 2011). En este contexto, la frontera comienza a ser ocupada y utilizada de modo diferente de lo que venía ocurriendo hasta entonces, pues el entorno constituido por el régimen militar fomentó una ampliación del número de trabajadores dependientes económicamente de la frontera, trabajadores no siempre naturales de la región y no conocedores del "saber pasar". La absolución de Rafael Barrios y el parecer del Ministerio Público sobre la cuestión contenida en el proceso criminal antes discutido, demuestra ello. La práctica desarrollada por el trabajador fue considerada culturalmente aceptada dentro de una coyuntura de escasez de mercancías, lo que imposibilitaría la condenación del acusado por el supuesto crimen de contrabando. El fortalecimiento del Estado nación a lo largo del siglo xx interfiere directamente en ello. No es posible afirmar que el "saber pasar" presente en la práctica y Barrios fue extinto o que él dejó de existir en la vida de los habitantes de la frontera Brasil-Paraguay, pero, con seguridad, es posible pensar que ha perdido su inocencia. 
El mecanismo utilizado por el Estado brasileño para garantizar su soberanía en la región pasó por el acicate al crecimiento demográfico, por el desarrollo económico y por el control de la circulación de personas que eran contrarias al régimen civil-militar. En gran medida, estos aspectos fueron alcanzados con la construcción de la hidroeléctrica y con la concentración del poder político en los agentes vinculados a la dictadura. Así, no se observa en este periodo un fortalecimiento de los dispositivos estatales que tenían como objetivo fiscalizar y cohibir el contrabando y el descamino.

El impacto no "saber pasar" no es resultado de la persecución o de la represión del Estado, sino de la ampliación del número de personas dependientes del mercado paraguayo y de la alteración de las mercancías disponibles en Ciudad del Este, que fomentó una situación en que el "puente" de la frontera Brasil-Paraguay fuera utilizado por la población local en el ejercicio de sus prácticas tradicionales y en el mantenimiento de sus redes trasnacionales, pero también por compradores interesados en adquirir productos importados con precios atractivos y por los primeros "sacoleiros", que en aquel momento se responsabilizaban por la compra, distribución y reventa, principalmente, de ropas, bebidas y cigarrillos falsificados.

Nace el imaginario que vincula a Paraguay a la piratería y nace un contexto donde las prácticas tradicionales van perdiendo la importancia y la visibilidad. El mercado paraguayo comienza a volverse atractivo y a recibir más visitantes, adquiriendo la fama por el comercio y encubriendo con una niebla, antiguos modos de vivir. Muchos de los habitantes tradicionales de la región de la frontera se insertarían en la lógica comercial impuesta por la adherencia entre el desempleo existente y las posibilidades que surgían con el fortalecimiento de la zona de libre comercio paraguaya (Catta, 2002), apareciendo una homogeneización de las prácticas sociales realizadas.

La circulación trasnacional tradicional en la frontera entre el Brasil y Paraguay no ha dejado de existir. Aún es común la presencia de redes sociales trasnacionales, el tránsito entre las fronteras con el objetivo de complementar los víveres y de utilizar servicios existentes en una $u$ otra municipalidad fronteriza, así como la flexibilización de la autoidentificación de muchos habitantes. No obstante, el rápido crecimiento obtenido con la apertura de la Carretera Internacional entre el Puerto de Paranaguá, Brasil, hasta Asunción, Paraguay, y la construcción de la hidroeléctrica de Itaipú han contribuido con el cambio en el modo de uso de la frontera al insertarla de modo más directo en el proceso de circulación de mercancías, aprovechándose de la fuerza de trabajo excedente y de las políticas tributarias paraguayas.

\section{La frontera y el avance neoliberal}

La dictadura militar brasileña, asociada a la construcción de la hidroeléctrica, ha insertado un control político externo en la ciudad de Foz de Iguazú, restringiendo el espacio antes ocupado por las clases dominantes locales. Tal situación comenzó a ser modificada durante el proceso de democratización de Brasil, ocasión en que se buscó la construcción de una cohesión entre los diferentes intereses políticos y económicos de la ciudad por medio de una revalorización del sector turístico (Ribeiro, 2015). No obstante, en este momento histórico ocurrió de modo simultáneo al proceso de liberalización económica del país, que interfirió directa e indirectamente en los flujos comerciales en la frontera de Brasil con Paraguay durante la década de 1990. 
En un primer momento, se constata un cambio de perspectiva en aquello que se refiere a las estrategias de seguridad en las regiones de frontera. Con el propósito de romper con la militarización existente en las décadas anteriores, las políticas adoptadas tras la democratización partían del supuesto que la seguridad en la frontera debería estar asociada al desarrollo humano. De este modo, en vez de hacer inversión en la presencia de un contingente militar mayor en las fronteras, los esfuerzos fueron direccionados al empoderamiento de grupos de la sociedad civil, externalizando la responsabilidad en aquello que se refiere al desarrollo regional. Así, el control aduanero se volvió aún más frágil, facilitando la actuación de los circuitos de compra y venta de mercancías ilegales y también la evasión fiscal (Cardin, 2012).

La mayor porosidad de los límites territoriales fue acompañada de diferentes cambios económicos que contribuyeron con el fortalecimiento del "circuito sacoleiro" como, por ejemplo, los cambios en los procesos de exportación e importación, los acuerdos comerciales entre las naciones, la reestructuración productiva y el largo periodo de recesión económica vivida por el país. Tales factores, asociados con elementos locales, como el fin de la construcción de la Usina de Itaipu, promocionaron el surgimiento de una inmensa masa de desempleados y una configuración tributaria atractiva para la práctica del contrabando y del descamino. En este contexto, se concluye que el "circuito sacoleiro" es derivado, principalmente, de la coexistencia de tres elementos: fuerza de trabajo excedente en el mercado laboral brasileño, fragilidad en el control aduanero y disparidades tributarias entre los países vecinos (Cardin, 2011).

Como hemos visto, la circulación de mercancías entre Brasil y Paraguay es una práctica antigua, anterior a la presencia efectiva de los dispositivos del estado en esta región de confluencia de las fronteras. No obstante, el cambio en el perfil de las mercancías negociadas y también en la intensidad de la presencia estatal ha alterado los flujos de esta circulación a lo largo del siglo xx. Hasta la década de 1960, los flujos eran concentrados en recursos naturales, principalmente la madera y yerba mate. Posteriormente, tenemos el tránsito de productos agrícolas como el café y la soja. La circulación de mercancías manufacturadas e industrializadas ocurría en menor número y con finalidad de suministro local. A partir de la década de 1960, los productos agrícolas continúan siendo visualizados, pero con la presencia del contrabando de whisky, además de los productos de higiene y alimentos. Con el fortalecimiento de la zona de libre comercio en Paraguay en la década de 1970, se observa una mayor diversidad de productos y un aumento en el flujo de compradores (Fiorotti, 2015).

Sin embargo, es durante la década de 1990 que se visualiza el ápice de este mercado. Los productos electrónicos de origen asiático, elaborados con bajo costo, comienzan a ser vendidos por trabajadores extranjeros que no poseen ninguna seguridad laboral en las tiendas y carpas de Ciudad del Este, Paraguay. Productos que eran comprados y puestos a disposición por miles de trabajadores brasileños excluidos del mercado laboral nacional, pero que veían en el circuito sacoleiro un camino más corto para la obtención de una renta mínima o aun mismo para obtención de recursos muy superiores a aquellos disponibles en los empleos considerados formales.

En este periodo, categorías como pasero, mulas, mesiteros, cuota, arbolito, trabajo hormiga, comienzan a volverse usuales en la región. En determinado momento, cerca de $60 \%$ de los trabajadores económicamente activos de Foz de Iguazú dependían directa e indirectamente del comercio con Paraguay (Cardin, 2011). Situación que hizo que el Puente de la Amistad adquiriera características de un puente urbano, 
debido al creciente número de coches y personas que transitaban sobre él diariamente. En medio de eso, pocas detensiones eran realizadas debido a la falta de agentes aduaneros y también debido a la dificultad en calcular la relación entre la legalidad y la legitimidad de tales prácticas. Se creía además que la entrada de mercancías importadas auxiliaría en el equilibrio de los precios en el mercado brasileño y en la ampliación del consumo.

En medio de acercamientos y alejamientos en el modelo de gestión adoptado por el Partido da Social Democracia Brasileira (PSDB) y el Partido do Trabalhadores (PT) en el gobierno federal, el primer mandato del PT presentó diferencias significativas en lo que se refiere a la presencia del Estado en las fronteras brasileñas, permitiendo un mayor control de evasión fiscal y un rompimiento con los circuitos de tráfico de drogas y armas que abastecen los grandes centros brasileños, muchas aduanas fueron reconstruidas y el contingente de fiscales y policías federales ampliado. Tal situación ha provocado reacciones inmediatas en la configuración del circuito sacoleiro y en las relaciones sociales que involucraba, haciendo que Foz de Iguazú tuviera un periodo de crisis y de reorganización económica y social en la primera década del siglo xxi (Cardin, 2015).

El éxito de las Unidades de Policía Pacificadora implantadas en Río de Janeiro, programa de seguridad que visaba la ocupación de las zonas de conflicto por parte del Estado en una dimensión todavía más amplia, la disminución de la criminalidad en las ciudades que realizarían la Copa de las Confederaciones, la Copa del Mundo de Fútbol, los Juegos Olímpicos y Paraolímpicos, exigieron un control riguroso de las "puertas" de acceso a Brasil. En este contexto fueron realizadas innumerables operaciones especiales de seguridad en toda franja de frontera del país con la intención de limitar la entrada de armas y drogas, debilitando a los grupos criminales. Foz del Iguazú ha sentido directamente el impacto de tales políticas. La recesión económica, la disminución de la circulación de compradores y el aumento de la violencia en la municipalidad durante la primera década del siglo xxi son elementos que, de algún modo, fueron influenciados por las políticas de control de flujos (Cardin, 2015).

La inauguración de la nueva aduana en la frontera de Foz de Iguazú-Brasil y Ciudad del Este-Paraguay dificultó la utilización del Puente de la Amistad como vía de salida de las mercancías adquiridas en el microcentro paraguayo. La respuesta inmediata del circuito sacoleiro fue la utilización de puertos, que no eran oficiales o reglamentados, para realizar la travesía de las mercancías. Algunos de estos puertos ya existían y eran utilizados por el tráfico de drogas y armas, pero muchos otros surgieron. La diferencia entre pasar la mercancía por una vía pública (Puente de la Amistad) o pasar la mercancía por un espacio muchas veces "privado" (los puertos) ha fomentado disputas entre diferentes grupos, poniendo la municipalidad entre las diez ciudades más violentas de Brasil en los años de 2006 y 2007 (Cardin, 2013).

Mientras las relaciones comerciales entre Foz de Iguazú y Ciudad del Este comenzaban a ser más controladas, la circulación de trabajadores y mercancías en la frontera de Guaíra y Salto del Guairá se fortalecía, pues allá el control todavía no se presentaba tan riguroso comparado con Foz de Iguazú (Fiorotti, 2015). No obstante, no es sencillo afirmar que hubo una migración inmediata de parte del circuito sacoleiro, el entendimiento de esto involucra la observación de otros movimientos. Supuestamente, con la ampliación de la utilización de los puertos en la región de Foz de Iguazú, la Hacienda y la Policía Federal hicieron inversión en tecnología (aviones no tripulados y barcos) para monitorear el Lago de Itaipú, haciendo migrar los puertos 
clandestinos a otros puntos de la frontera fluvial, llevando consigo parte de la violencia existente en Foz de Iguazú (Cardin, 2015).

Así, se constata que el número de homicidios ocurridos en Foz de Iguazú viene bajando sucesivamente tras 2008, mientras que los indicadores de otras municipalidades que hacen frontera con Paraguay se alzan. En fin, la modificación en la fiscalización impuesta por el gobierno brasileño ha exigido una reorganización del circuito sacoleiro, visualizada: 1) en la disminución de los grupos que actúan en Foz de Iguazú y, consecuentemente, en la disminución de la violencia en la municipalidad; 2) en la pulverización de los puertos por toda la región del Lago de Itaipú y; 3) en el fortalecimiento de las relaciones comerciales entre Guaíra, Brasil, y Salto del Guairá, Paraguay, para atender compradores y grupos de mulas "sacoleiros" que perdieron espacio en la utilización de los lugares de paso existentes anteriormente.

\section{Reflexión final}

Dentro del recorte histórico analizado, se observa que los usos de la frontera por los habitantes de la región se relacionan con el fortalecimiento de la presencia estatal y con la expansión capitalista para el interior de Brasil. Mientras los dispositivos estatales no ejercían rigurosamente sus funciones y el capitalismo vivía una fase de acumulación primitiva, con expropiación directa de los recursos naturales y de los trabajadores sin mediación legal, los habitantes locales ocupaban y vivían en la región sin considerar los límites jurídicos. La libre circulación y las redes trasnacionales hacían parte de lo cotidiano y garantizaban la existencia de modos de vivir vinculados a una territorialidad propia.

Durante el siglo $\mathrm{xx}$ se visualiza el intento del Estado nación en ocupar y nacionalizar la región, primeramente por medidas administrativas y de poblamiento y, posteriormente, con la presencia militar. El propósito de tales políticas era buscar la concretización de la "buena frontera", o sea, el rompimiento geográfico, político y cultural que marcaría el límite territorial entre dos naciones. Para tanto, se buscaba el fortalecimiento de la identidad nacional, desconsiderando el contínuum colonial y las redes trasnacionales existentes, así como la inserción de la región en la lógica política y administrativa ejercida por el gobierno federal.

Por fin, con el proceso de democratización y de apertura económica del país en el final de la década de 1980, se constata un rápido crecimiento del comercio en la región y la proliferación de ocupaciones que, de distintas formas, buscaban aprovechar la asimetría económica y tributaria existente entre Brasil y Paraguay. En este contexto, otros fenómenos sociales ganaron visibilidad, encubriendo y modificando las prácticas sociales tradicionales de habitar en la frontera. Si en el pasado el territorio podría ser pensado por medio de su valor de uso, con el fortalecimiento del Estado y la expansión del capital en la región, cada vez más, se resalta su valor de cambio.

Actualmente, el circuito sacoleiro viene presentando diferentes facetas. Si durante la década de 1990 era explícito, expreso por convoyes que llegaban a unir más de cien colectivos alquilados para transportar mercancías de Paraguay, hoy es menos visible. La principal mercancía negociada en la frontera del Brasil con Paraguay es el cigarrillo, que entra en el territorio brasileño vía puertos no oficiales, pero, principalmente, por la frontera seca existente en los límites de Mato Grosso do Sul con Paraguay. 
Si un día Paraguay tuvo su importancia cimentada en la venta y distribución de electro electrónicos, eso ha bajado mucho en los últimos años debido a la facilidad de crédito en Brasil, el circuito de compras Brasil-Miami, EE.UU. y también por el surgimiento de otras formas de distribución de las mercancías. Aunque sean necesarias más investigaciones, las observaciones realizadas señalan un cambio en las relaciones comerciales establecidas en la frontera. Actualmente, se visualiza de un lado la inserción del circuito sacoleiro en el "mundo virtual", con la utilización de Internet para la realización de compras y para las transacciones bancarias, y del otro el regreso de un perfil de comprador típico de la década del 1980, con la presencia de trabajadores responsables por hacer directamente la mediación de las tiendas del Paraguay y el consumidor inmediato.

Hoy la frontera posee múltiples haceres, que son definidos por el lugar de origen del observador y también por la forma del uso atribuida a ella. Mientras los diferentes trabajadores insertos en el circuito sacoleiro ven en ella el potencial de lucro o de una renta mínima, otras personas la consideran como un mecanismo que ayuda a la disminución de los costos de la casa al ampliar la competencia de venta de productos esenciales, otros como una ampliación de las posibilidades de entretenimiento y, por fin, otros que son indiferentes a todo ello, indiferentes por no considerar la frontera como un límite.

\section{Referencias}

Albuquerque, J. L. C. (2010). A dinâmica das fronteiras: Os brasiguaios na fronteira entre o Brasil e o Paraguai. São Paulo, Brasil: Annablume.

Arriaga-Rodríguez, J. C. A. y Visintin, A. Z. (2012). Fronteras, por George Nathaniel Curzon. Una lectura crítica. México: Universidad de Quintana Roo.

Auto criminal 6 de abril de 1961. (Archivo NDP: 164/15, hoja 38). Análisis del Ministerio Público con relación al proceso siendo encaminado ao Juez de Derecho.

Brito, J. M. de (2005). Descoberta de Foz do Iguaçu e fundação da Colônia Militar. Paraná, Brasil: Travessa dos Editores.

Camal-Cheluja, T. L., Arriaga-Rodríguez, J. C. y Cardin, E. G. (2015). Fronteras y dinámicas transfronterizas en América Latina. Chetumal, México: Universidad de Quintana Roo.

Cardin, E. G. (2011). Laranjas e sacoleiros: Um estudo da precarização do trabalho no capitalismo contemporâneo. Cascavel, Brasil: Editora da Universidade Estadual do Oeste do Paraná.

Cardin, E. G. (2012). O Governo Brasileiro nos processos de demarcação e defesa das fronteiras Sulamericanas. Memorias del XIII Seminario Internacional de Verano. Caribe: Economía, Política y Sociedad. Universidad Quintana Roo, Chetumal.

Cardin, E. G. (2013). Mecanismos de contrabando y tráfico en la Triple Frontera. En F. Carrión y V. Llugsha, Fronteras: Rupturas y convergencias. (pp. 255-266). Quito, Ecuador: Flacso.

Cardin, E. G. (2015). A Expansão do Capital e as Dinâmicas das Fronteiras. Jundiaí, Brasil: Paco.

Catta, L. E. (2002). O cotidiano de uma fronteira: A pervesidade da modernidade. Cascavel, Brasil: Edunioeste. 
Colodel, J. A. (1988). Obrages E companhias colonizadoras: Santa Helena na história do oeste paranaense até 1960. Santa Helena, Brasil: Prefeitura Municipal.

De Souza, A. D. (2009). Formação econômica e social de Foz do Iguaçu: um estudo sobre as memórias constitutivas da cidade (1970-2008) (Tesis de doctorado). Universidade de São Paulo, Brasil.

De Souza, E. B. C. (2011). A geopolítica da produção do espaço: Localização da hidrelétrica da Itaipu Nacional. Revista Geografares, (9), 141-167.

Dorfman, A. (2009). A cultura do contrabando e a fronteira com um lugar de memória. Estudios Históricos, (01).

Espósito, T. (2013). As relações Brasil-Paraguai: Do litígio da fronteira brasileiro-paraguaia (1962) à Ata das Cataratas (1966). Revista de Estudos e Pesquisas sobre as Américas, 7(1) 33-49.

Fiorotti, C. (2009). Mudanças no mundo dos trabalhadores: Um estudo sobre as vendedoras de produtos por catálogo avon e natura (Tesis de maestría). Universidade Estadual do Oeste do Paraná.

Fiorotti, C. (2015). Os descaminhos na fronteira: Fiscais do estado e trabalhadores nos processos de apreensão de mercadorias na fronteira Brasil-Paraguai (19802015). Revista Ambivalências, 3(5), 85-114.

Freitag, L. da C. (2001). Fronteiras perigosas: Migração e brasilidade no extremo-oeste paranaense (1937 - 1954). Cascavel, Brasil: Edunioeste.

García, N. (1990). Culturas híbridas. Estrategias para entrar y salir de la modernidad. México: Grijalbo.

García, N. (2000). ¿De qué lado estás? Metáforas de la frontera México-Estados Unidos. En A. Grimson, Fronteras, naciones e identidades: La periferia como centro (pp. 139-151). Buenos Aires, Argentina: Ciccus.

Garcia, N. J. (1999). Estado Novo, ideologia e propaganda política. Recuperado de http:/ / www.ebooksbrasil.org/adobeebook/estadonovo.pdf

Giménez, V. y Montenegro, S. (2006). La triple frontera: Globalización y construcción social del espacio. Buenos Aires, Argentina: Miño y Dávila.

Godinho, P. (2009). Desde a idade de seis anos, fui muito contrabandista - O concelho de Chaves e a Comarca de Verín, entre velhos quotidianos de fronteira e novas modalidades emblematizantes. En D. Freire, E. Rovisco e I. Fonseca (Coords.), Contrabando na Fronteira Luso-Espanhola (pp. 29-56). Lisboa, Brasil: Edições Nelson de Matos.

Grimson, A. (2005). Cortas puentes, cortar pollos: conflictos económicos y agencias políticas em Uruguayana (Brasil) - Libres (Argentina). En R. C. de Oliveira y S. G. Baines (Orgs.), Nacionalidade e etnicidade em fronteiras (pp. 21-53). Brasília, Brasil: Universidad de Brasilia.

Hirata, D. (2014). Ilegalismos. En R. S. de Lima, J. L. Ratton y R. G. Azevedo (Orgs.), Crime, polícia e justiça no Brasil (pp. 97-104). São Paulo, Brasil: Contexto.

Ingold, T. (2015). Estar vivo: Ensaios sobre movimento, conhecimento e descrição. Petrópolis, Brasil: Editora Vozes.

Lima, P. (2001). Foz do Iguaçu e sua história. Foz do Iguaçu, Perú: Editora do Autor.

Montenegro, S. y Giménez, V. (2010). La triple frontera: Dinámicas culturales y procesos transnacionales. Buenos Aires, Argentina: Espacio. 
Renoldi, B. (2014). Tus amigos son mis amigos: Agentes y Estado en un paso de frontera argentino. Alteridades, 24(47), 89-100.

Ribeiro, D. G. (2015). Metamorfoses na cidade: Tensões e contradições na produção e apropriação do espaço urbano em Foz do Iguaçu (Tesis de maestría). Toledo, Paraná: Unioeste.

Simmel, G. (1996). A Ponte e a Porta. Revista Política e Trabalho, (12), 10-14.

Sperança, A. (1992). Cascavel, a história. Curitiba, Brasil: Lagarto Editores.

Valdebenito, F. y Lube, M. (2014). As fronteiras da modernidade. O espaço tacno-ariquenho e a nacionalização do norte grande chileno (1883-1929). Estudos Ibero-Americanos, 40(2), 277-303.

Wachowicz, R. C. (1982). Obrages, mensus e colonos-história do oeste Paranaense. Curitiba, Brasil: Editora Vicentina.

Zaidenwerg, C. (2013). "Amar, honrar y servir a la patria”. Las escuelas del territorio rionegrino y su aporte a la obra argentinizadora en el Sur (1908-1930). Revista Digital de la Escuela de Historia, 5(9), 61-89.

Eric Cardin

Brasileño. Doctor en Sociología por la Universidad Estadual Paulista (UnEsp-Brasil). Postdoctor en Antropología Social por la Universidad Nacional de Misiones (UnAMArgentina). Profesor de la Universidad Estadual del Oeste de Paraná (Unioeste-Brasil). Líneas de investigación: trabajo, prácticas ilegales y flujos en regiones de frontera. Algunas de sus publicaciones más recientes: Cardin, E. G. y Albuquerque, J. L. (2018). Fronteiras e Deslocamentos. Revista Brasileira de Sociologia, 06(12), 115-131 y Cardin, E. G. (2016). Ituzaingó não é Winston Parva ou como os permanentes se tornar outsiders. Revista Ambivalências, 4(8), 213-242.

Cíntia Fiorotti Lima

Brasileña. Doctora en Historia por la Universidad Federal de Uberlândia (Ufu-Brasil). Profesora del cuadro propio de la Secretaría de Educación del Estado de Paraná (SEED-Brasil). Líneas de investigación: trabajo, prácticas ilegales y flujos en regiones de frontera. Publicaciones más recientes: Fiorotti, C. (2015). Os descaminos na frontera: Fiscais do estado e trabalhadores nos procesos de apreensão de mercadorias na frontera Brasil-Paraguai (1980-2015). Revista Ambivalências, 3(5), 85-114 y Fiorotti, C. (2014). Migrações e mudanças no mundo dos trabalhadores a partir das trajetórias ocupacionais de trabalhadores em Guaíra-PR entre 1970 e 2010. Revista Tempo da Ciência, 21(42), 33-44. 